\title{
Development and Validation of a Specific Stability Indicating High Performance Liquid Chromatographic Methods for Related Compounds and Assay of Solifenacin Succinate
}

\author{
B. V. Rami Reddy, ${ }^{1,2}$ B. Srinivasa Reddy, ${ }^{2}$ N. V. V. S. S. Raman, ${ }^{2}$ \\ K. Subhash Reddy, ${ }^{2}$ and C. Rambabu ${ }^{1}$ \\ ${ }^{1}$ Department of Chemistry, Acharya Nagarjuna University, Nagarjunanagar, Guntur 522210, Andhra Pradesh, India \\ ${ }^{2}$ Hetero Drugs Limited, Balanagar, Hyderabad 500018, Andhra Pradesh, India
}

Correspondence should be addressed to C. Rambabu; rbchintala@gmail.com

Received 12 June 2012; Accepted 18 October 2012

Academic Editor: Ahmet Kilic

Copyright (C) 2013 B. V. Rami Reddy et al. This is an open access article distributed under the Creative Commons Attribution License, which permits unrestricted use, distribution, and reproduction in any medium, provided the original work is properly cited.

Gradient, reverse phase liquid chromatographic methods were developed separately for the related compounds and solifenacin succinate, an active pharmaceutical ingredient used for the treatment of overactive bladder. Gradient LC method was employed for related compounds. The mobile phase-A contains a $0.01 \mathrm{M}$ phosphate buffer $\mathrm{pH}: 3.5 \pm 0.05$ with orthophosphoric acid (88\%) and mobile phase-B contains a mixture of acetonitrile and water in the ratio of $90: 10(\mathrm{v} / \mathrm{v})$. The flow rate was $1.0 \mathrm{~mL} / \mathrm{minute}$, column temperature was kept at $35^{\circ} \mathrm{C}$, and detection was monitored at $220 \mathrm{~nm}$. In the developed HPLC method the resolution between solifenacin succinate and its closely eluting impurity, that is, solifenacin $\mathrm{N}$-oxide was found to be greater than 3.0. The drug was subjected to stress conditions such as hydrolysis, oxidation, photolysis, and thermal degradation. Considerable degradation was found to occur in only oxidative stress condition. Degradation product formed during oxidative stress condition was found to be impurity-C and it can be identified by LC-MS. The stress samples were assayed against a qualified reference standard and the mass balance was found close to $99.5 \%$. The developed RP-LC method was validated as per ICH guidelines. We also developed LC-MS/MS method for determination and identification of these impurities in solifenacin succinate.

\section{Introduction}

Muscarinic receptors play an important role in several major cholinergically mediated functions, including contractions of urinary bladder smooth muscle and stimulation of salivary secretion. The muscle in the wall of the bladder is called the detrusor muscle. It can sometimes contract in uncontrollable spasms, and this is often referred to as having an overactive bladder. The anticholinergic agents are widely used for the treatment of overactive bladder [1]. Solifenacin succinate is a muscarinic receptor antagonist belongs to anticholinergics and has higher selectivity for the urinary bladder over salivary glands $[2,3]$. It acts as a selective antagonist to the $\mathrm{M}(3)$ receptor and works by relaxing the bladder muscles to prevent urgent, frequent, or uncontrolled urination. Chemically, solifenacin succinate is butanedioic acid, compound with (1S)-(3R)-1-azabicyclo [2.2.2] oct-3-yl 3,4-dihydro-1-phenyl-2 $(1 \mathrm{H})$-iso-quinolinecarboxylate $(1: 1)$ having an empirical formula of $\mathrm{C}_{23} \mathrm{H}_{26} \mathrm{~N}_{2} \mathrm{O}_{2} \mathrm{C}_{4} \mathrm{H}_{6} \mathrm{O}_{4}$ and a molecular weight of 480.55 .

Solifenacin succinate (Figure 1) is a white to paleyellowish-white crystal or crystalline powder. It is freely soluble at room temperature in water, glacial acetic acid, dimethyl sulfoxide, and methanol. Solifenacin succinate is available in the market under the brand name of VESIcare in the form of tablets. It is available in two dosage strengths, that is, $5 \mathrm{mg}$ or $10 \mathrm{mg}$ and is formulated for oral administration. In addition to the active ingredient solifenacin succinate, each VESIcare tablet also contains the following inert ingredients: lactose monohydrate, corn starch, hypromellose 2910, magnesium stearate, talc, polyethylene glycol 8000 and titanium dioxide with yellow ferric oxide (5 mg VESIcare tablet), or red ferric 
(1S)-3, 4-dihydro-1-phenyl-2 (1H)-isoquinolinecarboxylic acid (3R)-1-azabicyclo [2.2.2] oct-3-yl

ester, succinate (solifenacin succinate).<smiles>O=C(O)CCC(=O)O</smiles>

1-phenyl-3, 4-dihydro isoquinoline (impurity-A)<smiles>c1ccc(C2=NCCc3ccccc32)cc1</smiles>

(1S)-1-phenyl-1, 2, 3, 4-tetrahydro isoquinoline (impurity-B)<smiles>c1ccc([C@H]2NCCc3ccccc32)cc1</smiles><smiles>O=C(O[C@H]1CN2CCC1CC2)N1CCc2ccccc2C1c1ccccc1</smiles>

(1S)-3,4-dihydro-1-phenyl-2-(1H)-isoquinolinecarboxylic acid (3R)-1-azabicyclo[2.2.2] oct -3-yl ester N-oxide (Impurity-C)

FIGURE 1: Chemical structures of solifenacin succinate, impurity-A, impurity-B, and impurity-C.

oxide (10 mg VESIcare tablet). The recommended dose of VESIcare is $5 \mathrm{mg}$ once daily. If the $5 \mathrm{mg}$ dose is well tolerated, the dose may be increased to $10 \mathrm{mg}$ once daily. VESIcare should be taken with water and swallowed whole. VESIcare can be administered with or without food.

Angioedema of the face, lips, tongue, and/or larynx has been reported with solifenacin. In some cases angioedema occurred after the first dose. Cases of angioedema have been reported to occur hours after the first dose or after multiple doses. Angioedema associated with upper airway swelling may be life threatening. If involvement of the tongue, hypopharynx, or larynx occurs, solifenacin should be promptly discontinued and appropriate therapy and/or measures necessary to ensure a patent airway should be promptly provided. Anaphylactic reactions have been reported rarely in patients treated with solifenacin succinate. Solifenacin succinate should not be used in patients with a known or suspected hypersensitivity to solifenacin succinate. In patients who develop anaphylactic reactions, solifenacin succinate should be discontinued and appropriate therapy and/or measures should be taken.

Solifenacin succinate is principally (98\%) bound to $\alpha 1$ acid glycoprotein's of human plasma and is highly distributed to non-CNS tissues, having a mean steady-state volume of distribution of $600 \mathrm{~L}$. The elimination half-life of solifenacin following chronic dosing is approximately 45-68 hours. Solifenacin is a substrate for cytochrome P450 enzyme CYP3A4 and extensively metabolized in the liver. Inducers 
TABLE 1: Gradient programme for related compounds.

\begin{tabular}{lcccc}
\hline Time (minutes) & Flow rate (mL/minute) & Mobile phase-A (\%) & Mobile phase-B (\%) & Gradient \\
\hline 0.01 & 1.0 & 80 & 40 & Equilibration \\
20 & 1.0 & 60 & 40 & Linear gradient \\
30 & 1.0 & 60 & 20 & Isocratic \\
32 & 1.0 & 80 & 20 & Reequilibration \\
40 & 1.0 & 80 & & Equilibration \\
\hline
\end{tabular}

TABLE 2: Gradient programme for assay.

\begin{tabular}{lcccc}
\hline Time (minutes) & Flow rate (mL/minute) & Mobile phase-A (\%) & Mobile phase-B (\%) & Gradient \\
\hline 0.01 & 1.0 & 70 & 30 & 60 \\
7 & 1.0 & 40 & 30 & Equilibration \\
10 & 1.0 & 70 & 30 & Reequilibration \\
15 & 1.0 & 70 & Equilibration \\
\hline
\end{tabular}

TABLE 3: System suitability report in related compounds method.

\begin{tabular}{lc}
\hline Compound name & USP resolution \\
\hline Between impurity-A and impurity-B & 8.53 \\
Between solifenacin and impurity-C & 3.62 \\
\hline
\end{tabular}

or inhibitors of CYP3A4 may alter solifenacin pharmacokinetics. Administration of $10 \mathrm{mg}$ VESIcare in the presence of $400 \mathrm{mg}$ of ketoconazole, a potent inhibitor of CYP3A4, the mean Cmax and AUC of solifenacin increased by 1.5 and 2.7-fold, respectively. Therefore, it is recommended not to exceed $5 \mathrm{mg}$ daily dose of VESIcare when administered with therapeutic doses of ketoconazole or other potent CYP3A4 inhibitors.

The primary metabolic routes of solifenacin are through $\mathrm{N}$-oxidation of the quinuclidin ring and 4R-hydroxylation of tetrahydroisoquinoline ring. One pharmacologically active metabolite (4R-hydroxy solifenacin), occurring at low concentrations and unlikely to contribute significantly to clinical activity, and three pharmacologically inactive metabolites ( $\mathrm{N}$-glucuronide and the $\mathrm{N}$-oxide and 4R-hydroxy-Noxide of solifenacin) have been found in human plasma after oral dosing.

Stability testing is performed during drug development to provide evidence of how the quality of a drug substance or drug product changes over time in response to a variety of environmental factors. Factors such as temperature, humidity, and light are studied to establish shelf life for the drug products and recommended storage conditions or packing.

Solifenacin succinate is not appearing in any pharmacopeia and no literature is available for determination of all process-related compounds and degradants in bulk drugs/pharmaceutical ingredient (API). Only few high performance liquid chromatography (HPLC) and mass spectroscopy methods were reported in literature for determination of solifenacin and related substances. High sensitive and rapid LC-electrospray ionization-MS-MS (in human plasma) and semi-micro-HPLC (in rat plasma) methods were reported for the determination of solifenacin succinate and its major metabolites in biological samples [4-6]. Ultrafast liquid chromatography for determination of related substances in solifenacin succinate, HPLC method for determination of solifenacin in bulk formulations and stereo selective HPLC method to quantify all the stereoisomers of solifenacin succinate in drug products [7-10], spectrophotometric, and HPTLC methods [11-13] were reported.

However the reported methods cannot be used for determination of all process-related compounds and degradation products, particularly solifenacin N-Oxide (Impurity-C) and also observed blank interferences. As per the requirements of various regulatory authorities, the impurity profile study of drug substances and drug products has to be carried out using a suitable analytical method in the final product [14, 15].

The main aim of this work was to develop a specific, accurate, and stability indicating gradient RP-HPLC method for the determination of all process-related compounds, degradation products, and assay in presence of its degradation products without any blank interferences. The developedrelated compounds and assay methods were validated as per the International Conference on Harmonization (ICH) guidelines [16]. Stability testing of new drug substances requires the stress testing to carry out and to elucidate the inherent stability characteristic of the active substance [17]. The advantages of the methods presented here are that they are simple, rapid, and stability indicative.

\section{Experimental}

2.1. Chemicals. Samples of solifenacin succinate and its three-related compounds, namely, impurity-A, impurity-B, and impurity-C were received from analytical research and Development Department of Hetero Research Foundation, Hyderabad, India. HPLC grade acetonitrile was purchased from Merck, Darmstadt, Germany. Analytical reagent grade potassium dihydrogen orthophosphate and orthophosphoric acid (88\%) were purchased from Qualizens fine chemicals, 


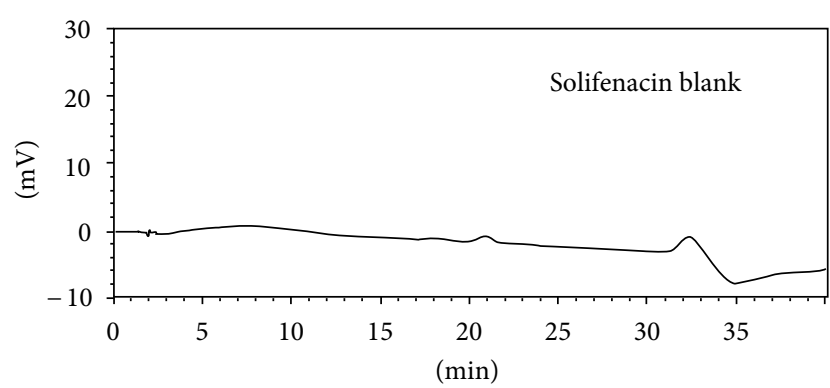

FIgURE 2: Typical HPLC chromatogram of blank solution.

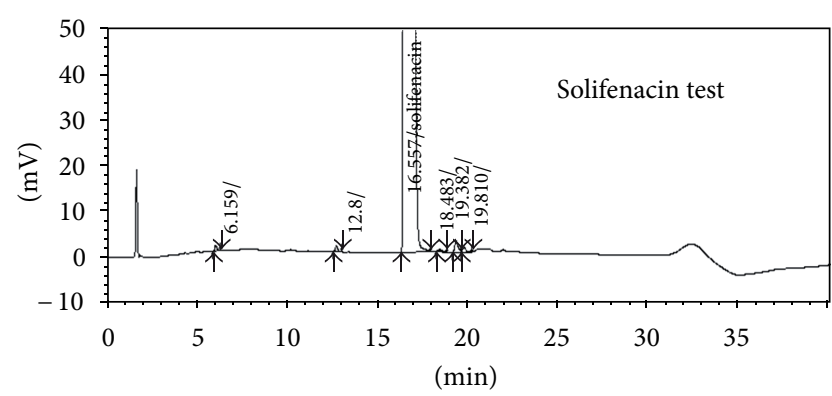

FIgURE 3: Typical HPLC chromatogram of pure solifenacin succinate.

Mumbai, India. High pure water was prepared by using Millipore Milli “Q” plus purification system.

2.2. Equipment. A Waters Alliance HPLC system equipped with 2695 separation module with quaternary gradient pumps with inbuilt auto injector, 270852 thermostatic compartment, and 2996 photo diode array detector was utilized for method development and validation which was located in Analytical Research and Development Department of Hetero Research Foundation (Lab-I), Hyderabad, India. Empower chromatography manager software (Waters) was used for data acquisition and system suitability calculations. Photo diode array detector was used for determining peak purity. The second instrument LC system consisted of quaternary gradient pump, auto sampler, column oven, and a UV-Visible dual wavelength detector. The output signal was monitored and integrated using LC Solutions chromatography Manager Software (2010 series HPLC, Shimadzu, Japan).

Shimadzu HPLC system was utilized in ruggedness study which was located in Analytical Research and Development Department of Hetero Research Foundation, Hyderabad, India (Lab-II).

2.3. Chromatographic Conditions. The chromatographic separation was achieved on waters symmetry shield RP-18 $150 \mathrm{~mm} \times 4.6 \mathrm{~mm}, 5 \mu \mathrm{m}$ column by performing gradient programme. The gradient HPLC method for related compounds employs variable solutions of mobile phase-A and mobile phase- $\mathrm{B}$ used as mobile phase. The mobile phaseA contains $0.01 \mathrm{M}(1.36 \mathrm{~g}$ in $1000 \mathrm{~mL}$ of water) potassium dihydrogen orthophosphate, adjusted to $\mathrm{pH}: 3.5 \pm 0.05$ using

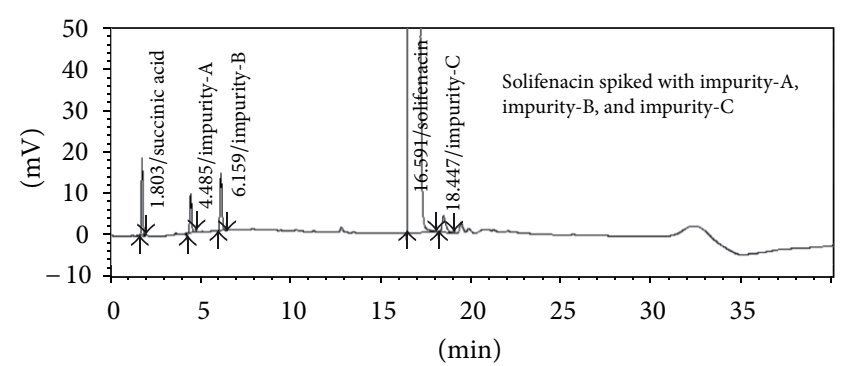

FIGURE 4: Typical HPLC chromatogram of pure solifenacin succinate spiked with $0.15 \%$ of impurity-A, impurity-B, and impurity-C.

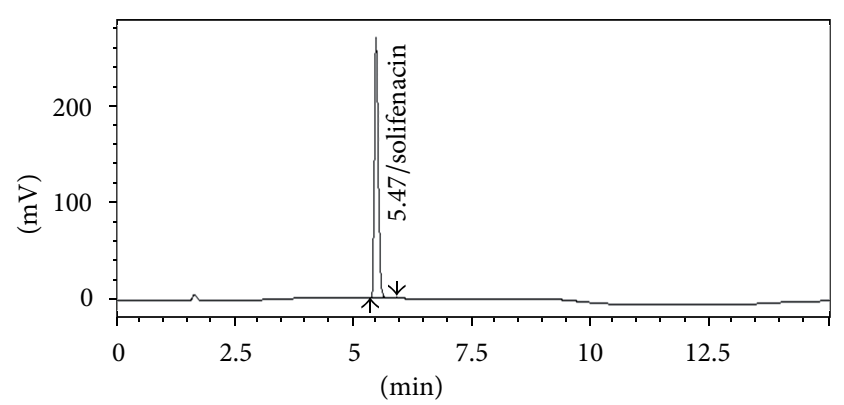

Figure 5: Typical HPLC chromatogram of solifenacin in assay method.

orthophosphoric acid (88\%) solution and mobile phase-B contains mixture of acetonitrile and water in the ratio of $90: 10(\mathrm{v} / \mathrm{v})$. The flow rate of the mobile phase was kept $1.0 \mathrm{~mL} / \mathrm{minute}$. The HPLC gradient programme performed for related compounds separation was shown in Table 1.

The column temperature was maintained at $35^{\circ} \mathrm{C}$ and the detector wavelength was monitored at a wavelength of $220 \mathrm{~nm}$. The injection volume was $10 \mu \mathrm{L}$ and mixture of mobile phase-A and acetonitrile in the ratio of $90: 10(\mathrm{v} / \mathrm{v})$ was used as diluent. To reduce the analysis time, gradient programme was modified for assay determination, and all other remaining chromatographic parameters kept same as related compounds method. The HPLC gradient programme performed for assay determination was shown in Table 2. All calculations concerning the quantitative analysis were performed with external standardization by measurement of peak areas.

\subsection{LC-MS Conditions}

Equipment. A Waters Alliance 2695 HPLC and Micromass ZQ-2000 MS (Waters Assoc., Milford, MA, USA.), controlled with Mass Lynx version 4.0 software, were used for detection. The analytical column was a symmetry shield RP$18150 \mathrm{~mm} \times 4.6 \mathrm{~mm}, 5 \mu \mathrm{m}$ used for separation of desired impurities. For mass spectroscopy detection, the parameters were checked by injecting spiked sample of solifenacin succinate and oxidative stressed sample. Mass spectrometer was 
TABLE 4: System suitability report in assay method.

\begin{tabular}{lccc}
\hline Compound name & USP resolution & Asymmetry factor for solifenacin & Theoretical plates for solifenacin \\
\hline Between solifenacin and impurity-C & 2.5 & 1.32 & 18664 \\
\hline
\end{tabular}

TABLE 5: Summary of recovery results.

\begin{tabular}{lcccc}
\hline \multirow{2}{*}{ Level } & \multicolumn{2}{c}{ \%Recovery range (related compounds method) } & \multicolumn{2}{c}{ \%Recovery range (assay method) } \\
& impurity-A & impurity-B & impurity-C & $99.6-100.4$ \\
\hline Level-1 & $97.87-100.0$ & $100.0-101.0$ & $102.55-104.08$ & $100.0-100.1$ \\
Level-2 & $102.56-104.13$ & $97.61-98.80$ & $103.81-104.63$ & $100.0-100.2$ \\
Level-3 & $99.50-101.42$ & $100.80-104.92$ & $102.59-105.31$ & \\
Level-4 & $101.9-103.41$ & $99.91-100.67$ & $101.06-103.94$ & \\
\hline
\end{tabular}

provided with electrospray ionization source and operating parameters were as follows.

LC-MS and Mass Spectrometric Conditions. The chromatographic separation was achieved on waters symmetry shield RP-18 $150 \mathrm{~mm} \times 4.6 \mathrm{~mm}, 5 \mu \mathrm{m}$ column by performing gradient programme. The gradient HPLC method employs variable solutions of mobile phase- $\mathrm{A}$ and mobile phase- $\mathrm{B}$ used as mobile phase. The mobile phase-A contains $0.01 \mathrm{M}$ ammonium formate in water, adjusted to $\mathrm{pH}: 3.5 \pm 0.05$ with formic acid solution and mobile phase- $\mathrm{B}$ contains mixture of acetonitrile and water in the ratio of $90: 10(\mathrm{v} / \mathrm{v})$. The flow rate of the mobile phase was kept $0.9 \mathrm{~mL} /$ minute. The HPLC gradient program for related compounds method was set as: time/\% mobile phase-B: $0.01 / 20,20 / 40,30 / 40$, and $32 / 20$ with a postrun time of 8 minutes. The HPLC column temperature was maintained at $35^{\circ} \mathrm{C}$ and the detector wavelength was monitored at $220 \mathrm{~nm}$. The injection volume was $20 \mu \mathrm{L}$. A mixture of mobile phase-A and acetonitrile in the ratio of $90: 10(\mathrm{v} / \mathrm{v})$ was used as diluent.

The mass spectrometer was operated in positive ion electrospray mode. The capillary sprayer voltage was $3.5 \mathrm{kV}$ and the sample cone voltage was $25 \mathrm{~V}$. The source temperature was $120^{\circ} \mathrm{C}$ and the desolvation temperature was $350^{\circ} \mathrm{C}$. The desolvation and cone gas flow rates were set to 100 and $650 \mathrm{~L} / \mathrm{hr}$, respectively. The acquisition mass range is $\mathrm{m} / \mathrm{z}$ $0-1000$ at $0.5 \mathrm{~s}$ per scan with a $0.1 \mathrm{~s}$ interscan delay. All mass spectra are acquired in centroid mode.

2.5. Preparation of Standard Solutions. A stock solution of solifenacin succinate $(5.0 \mathrm{mg} / \mathrm{mL})$ was prepared by dissolving appropriate amount in the diluent. Working solutions of $0.5 \mathrm{mg} / \mathrm{mL}$ and $0.1 \mathrm{mg} / \mathrm{mL}$ were prepared from above stock solution for related compounds determination and assay determination, respectively. A stock solution of impurity mixture (impurity-A, impurity-B, and impurity-C) at $0.03 \mathrm{mg} / \mathrm{mL}$ was also prepared in diluent.

2.6. Specificity. Specificity is the ability of the method to measure the analyte response in the presence of its potential impurities [18]. Stress testing of the drug substance can help identify the likely degradation products, which can in turn help establish the degradation pathways and the intrinsic stability of the molecule and validate the stability indicating power of the analytical procedures used. The specificity of the developed HPLC method for solifenacin succinate was determined in the presence of its impurities, namely, impurity- $\mathrm{A}$, impurity- $\mathrm{B}$, and impurity- $\mathrm{C}$ and degradation products. Forced degradation studies were also performed on solifenacin succinate to provide an indication of the stability indicating property and specificity of the proposed method. The stress conditions employed for degradation study included Light 1.2 million LUX hrs (carried out as per ICH Q1B), heat $\left(105^{\circ} \mathrm{C}\right)$, acid hydrolysis $(0.1 \mathrm{~N} \mathrm{HCl})$, base hydrolysis $(0.1 \mathrm{~N} \mathrm{NaOH})$, water hydrolysis, and oxidation $\left(10 \% \mathrm{H}_{2} \mathrm{O}_{2}\right)$. For heat and light studies, study period was two days whereas for acid, base, water hydrolysis, and oxidation, it was $4 \mathrm{hrs}$.

The photo degradation was carried out by exposing the solifenacin succinate sample in solid state to light providing an overall illumination of not less than 1.2 million lux hours and an integrated near ultraviolet energy of not less than $200 \mathrm{Wh} / \mathrm{m}^{2}$, which took about 10 days period in photo stability chamber. Stressed samples of solifenacin succinate generated were checked for peak purity of by using waters 2695 photo-diode array detector. The purity factor is within the threshold limit obtained in all stressed samples demonstrates the analyte peak homogeneity. Assay studies were carried out for stress samples against qualified reference standard and the mass balance (\%assay + \%impurities + $\%$ degradation products) was calculated. The assay was also calculated for bulk sample by spiking all three impurities (impurity-A, -B, and impurity-C) at the specification level (i.e., $0.15 \%$ of impurity- $\mathrm{A}, \mathrm{B}$, and $\mathrm{C}$ with respect to analyte concentration, which is $0.5 \mathrm{mg} / \mathrm{mL}$ ).

\subsection{Method Validation}

2.7.1. Precision. The system precision of the assay method was evaluated by carrying out five replicate injections of solifenacin succinate standard solution. The percentage of RSD was calculated for area of solifenacin peak from five replicate injections. The method precision of the assay method was evaluated by carrying out six independent assays of test sample of solifenacin succinate against qualified reference standard. The percentage of RSD of six assay values obtained was calculated. 
TABLE 6: Summary of robustness results.

\begin{tabular}{|c|c|c|c|c|}
\hline S. No. & Parameter & Variation & $\begin{array}{l}\text { Resolution between solifenacin } \\
\text { and impurity-C }\end{array}$ & $\begin{array}{l}\text { Resolution between impurity-A } \\
\text { and B }\end{array}$ \\
\hline \multirow{2}{*}{1} & \multirow{2}{*}{ Temperature $\left( \pm 5^{\circ} \mathrm{C}\right.$ of set temperature) } & (a) At $33^{\circ} \mathrm{C}$ & 3.8 & 8.7 \\
\hline & & (b) At $37^{\circ} \mathrm{C}$ & 3.4 & 8.1 \\
\hline \multirow{2}{*}{2} & \multirow{2}{*}{ Flow rate ( $\pm 20 \%$ of the set Flow) } & (a) At $0.8 \mathrm{~mL} / \mathrm{min}$ & 3.9 & 8.9 \\
\hline & & (b) At $1.2 \mathrm{~mL} / \mathrm{min}$ & 3.5 & 8.3 \\
\hline \multirow{2}{*}{3} & \multirow{2}{*}{$\mathrm{pH}( \pm 0.2$ units of set $\mathrm{pH})$} & (a) At 3.3 & 3.1 & 8.8 \\
\hline & & (b) At 3.7 & 3.3 & 8.6 \\
\hline
\end{tabular}

TABLE 7: Summary of forced degradation results.

\begin{tabular}{|c|c|c|c|c|}
\hline Stress condition & Time & $\begin{array}{l}\text { \%assay of active } \\
\text { substance }\end{array}$ & $\begin{array}{c}\text { Mass balance } \\
\text { (\%assay + \%impurities + } \\
\text { \%degradation impurities) }\end{array}$ & Remarks \\
\hline Acid hydrolysis $(0.1 \mathrm{~N} \mathrm{HCl})$ & $4 \mathrm{hrs}$ & 100.7 & 100.8 & No degradation is observed \\
\hline Base hydrolysis $(0.1 \mathrm{~N} \mathrm{NaOH})$ & $4 \mathrm{hrs}$ & 100.9 & 101.7 & No degradation is observed \\
\hline Oxidative $\left(10 \% \mathrm{H}_{2} \mathrm{O}_{2}\right)$ & $2 \mathrm{hrs}$ & 90.7 & 100.8 & $\begin{array}{l}\text { degraded into impurity-A and } \mathrm{C} \\
\text { and unknowns }\end{array}$ \\
\hline Water hydrolysis & $4 \mathrm{hrs}$ & 100.8 & 100.9 & No degradation is observed \\
\hline Thermal $\left(80^{\circ} \mathrm{C}\right)$ & 10 days & 100 & 100.1 & No degradation is observed \\
\hline Light (Photolytic) & 10 days & 100.3 & 100.4 & No degradation is observed \\
\hline
\end{tabular}

The system precision of the related compounds method was checked by injecting six replicate runs of solifenacin spiked with impurity blend solution (solifenacin $0.1 \%$ and each impurity $0.15 \%$ level with respect to test solution concentration, which is $0.5 \mathrm{mg} / \mathrm{mL}$ ). The percentage of RSD for area of each impurity- $A$, impurity- $B$, impurity-C, and solifenacin was calculated.

The method precision of the related compounds method was checked by injecting six individual test preparations and the percentage of RSD for each impurity-A, impurity-B, and impurity-C, was calculated. The intermediate precision of the method was also evaluated using different analysts and a different instrument in the same laboratory.

2.7.2. Limit of Detection (LOD) and Limit of Quantification (LOQ). The LOD and LOQ for solifenacin, impurity-A, impurity- $\mathrm{B}$, and impurity- $\mathrm{C}$ were estimated at a signal-tonoise ratio of $3: 1$ and $10: 1$, respectively, by injecting a series of dilute solutions with known concentration [19]. Precision study was also carried at the LOQ level by injecting six replicates of solifenacin spiked with impurity-A, impurity-B, and impurity-C blend and calculating the percentage of RSD of the peak areas.

2.7.3. Linearity. Linearity test solutions for assay method were prepared from stock solution at six concentration levels from 80 to $120 \%$ of assay analyte concentration $(80,90,100$, 110 , and $120 \%$ ). The peak area versus concentration data was performed by least-squares linear regression analysis. Linearity test solutions for related compounds method were prepared by diluting the impurity stock solution (2.5) to the required concentrations. The solutions were prepared at six concentration levels from LOQ to $150 \%$ of the specification level [LOQ, 0.075(50\%), 0.12(80\%), 0.135(90\%), 0.15(100\%), $0.18(120 \%)$, and $0.225(150 \%)]$.

2.7.4. Accuracy. The accuracy of the assay method was evaluated in triplicate at three concentration levels, that is, 80,100 , and $120 \%$ in bulk drug sample. The percentage of recoveries was calculated. Accuracy/recovery experiments were performed in triplicate. The bulk sample, provided by Hetero Research Foundation, does not show the presence of impurity-A and impurity-B. Some of the bulk samples show the presence of impurity-C in between 0.03 and $0.05 \%$ levels. Standard addition and recovery experiments were conducted to determine accuracy of the related compounds method for the quantification of all three impurities in bulk drug samples.

The accuracy study was carried out in triplicate at LOQ, $0.075 \%, 0.15 \%$, and $0.225 \%$ of the analyte concentration $(0.5 \mathrm{mg} / \mathrm{mL})$. The percentage of recoveries for impurity-A, impurity- $\mathrm{B}$, and impurity- $\mathrm{C}$ were calculated.

2.7.5. Robustness. To determine the robustness of the developed assay and related compound methods, experimental conditions were purposely altered and the resolution between solifenacin succinate and impurity-C, impurity-A, and impurity-B was evaluated. The flow rate of the mobile phase was $1.0 \mathrm{~mL} /$ minute. To study the effect of flow rate on the resolution, it was changed by 0.2 units, from 0.8 to $1.2 \mathrm{~mL} /$ minute, while other mobile phase components were held constant as stated in Section 2.3. The effect of change in the composition of organic modifier was checked by changing in the mobile phase composition. The effect of $\mathrm{pH}$ on resolution of impurities was studied by varying 


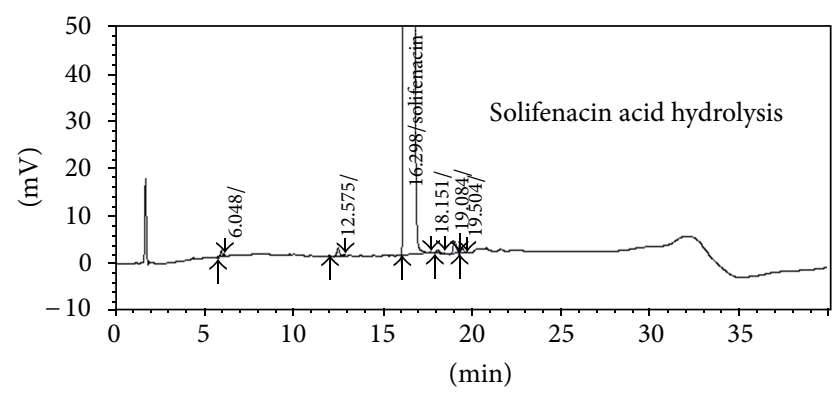

Figure 6: Typical HPLC chromatogram of $0.1 \mathrm{~N} \mathrm{HCl}$ stressed solution.

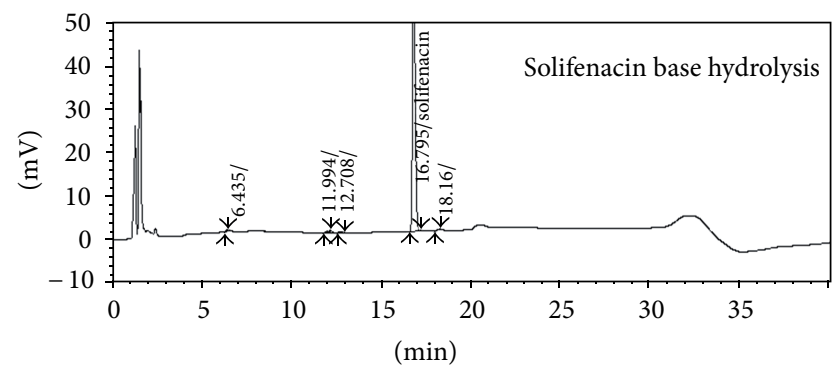

Figure 7: Typical HPLC chromatogram of $0.1 \mathrm{~N} \mathrm{NaOH}$ stressed solution.

$\pm 0.2 \mathrm{pH}$ units (at 3.3 and 3.7 buffer $\mathrm{pH}$ ). The effect of column temperature on resolution was studied at $33^{\circ} \mathrm{C}$ and $37^{\circ} \mathrm{C}$ instead of $35^{\circ} \mathrm{C}$. In all the above varied conditions, the components of the mobile phase were held constant as stated in Section 2.3.

2.7.6. Solution Stability and Mobile Phase Stability. The solution stability of solifenacin succinate in the assay method was carried out by leaving both the test solutions of sample and reference standard in tightly capped volumetric flasks at room temperature for $12 \mathrm{hrs}$ and $24 \mathrm{hrs}$ (for related compounds study period is $12 \mathrm{hrs}, 24 \mathrm{hrs}, 36 \mathrm{hrs}$, and $48 \mathrm{hrs}$ ). The same sample solutions were assayed for 6-hour interval up to the study period. The mobile phase stability was also carried out by assaying the freshly prepared sample solutions against freshly prepared reference standard solutions for 6-hour interval up to 24 hours. Mobile phase prepared was kept constant during the study period. The percentage recovery of assay of solifenacin succinate was calculated for the study period during mobile phase and solution stability experiments.

The solution stability of solifenacin succinate and its impurities in the related compounds method was carried out by leaving spiked sample solution in tightly capped volumetric flask at room temperature for 48 hours. Content of impurity- $\mathrm{A}$, impurity-B, and impurity- $\mathrm{C}$ was determined for every 12-hour interval up to the study period. Mobile phase stability was also carried out for 48 hours by injecting the freshly prepared sample solutions for every 12-hourinterval. Content of impurity-A, impurity-B, and impurity-C was checked in the test solutions. Mobile phase prepared was kept constant during the study period. The percentage of RSD for content of each impurity-A, impurity-B and impurity-C was calculated from initial, 12, 24, 36 and 48 hrs samples of the study period.

\section{Results and Discussion}

3.1. Method Development and Optimization. Impurity-C is the potential impurity present in bulk samples of solifenacin succinate. The main target of the chromatographic method is to get the separation of critical closely eluting impurities, namely, impurity-A and impurity-B, solifenacin, and impurity-C. Impurities were eluted very closely to each other by using different stationary phases like CN, C18, C8, and phenyl and different mobile phases containing buffers like phosphate, sulfate, and acetate with different $\mathrm{pH}$ (2.5-5) and using organic modifiers like acetonitrile and methanol in the mobile phase. $\mathrm{pH}$ of the buffer has played a significant role in achieving the separation between impurity-A and impurity$\mathrm{B}$, solifenacin and impurity-C.

The chromatographic separation was achieved on a waters symmetry shield RP-18, $150 \mathrm{~mm} \times 4.6 \mathrm{~mm}, 5 \mu \mathrm{m}$ column, by using variable mixtures of mobile phase-A and mobile phase- $\mathrm{B}$ as mobile phase. The mobile phase- $\mathrm{A}$ contains $0.01 \mathrm{M}$ potassium dihydrogen orthophosphate, $\mathrm{pH}$ adjusted to $3.5 \pm 0.05$ using orthophosphoric acid solution $(88 \%)$ and mobile phase-B contains a mixture of acetonitrile and water in the ratio of $90: 10(\mathrm{v} / \mathrm{v})$. The flow rate of the mobile phase was $1.0 \mathrm{~mL} /$ minute. The HPLC gradient program for related compounds method was set as time/\% mobile phase-B: $0.01 / 20,20 / 40,30 / 40$ and $32 / 20$ with a postrun time of 8 minutes. At $35^{\circ} \mathrm{C}$ column temperature, the peak shape of solifenacin succinate was found symmetrical. In the optimized conditions solifenacin succinate, impurity$A$, impurity-B, and impurity-C were well separated with a resolution of greater than 3 and the typical retention times of impurity-A, impurity-B, impurity- $\mathrm{C}$, and solifenacin succinate were about $4.1,6.1,8.2$, and 14.0 minutes, respectively. The system suitability results for related compounds method are given in Table 3 and the developed HPLC method was found to be specific for solifenacin succinate and its three related compounds, namely, impurity-A, impurity-B, and impurity-C. For the determination of assay of solifenacin succinate the HPLC gradient programme was set as time $/ \%$ mobile phase-B: $0.01 / 30,7 / 60$ and $10 / 30$ with a postrun time of 5 minutes. In the optimized assay method the resolution between solifenacin and impurity- $\mathrm{C}$ is greater than 2.5 and peak shape is symmetrical. The typical retention time of solifenacin is 6 minutes. The system suitability results for assay method were given in Table 4 and the developed method is specific for assay determination.

\subsection{Method Validation}

3.2.1. Precision. The $\%$ RSD for assay of solifenacin succinate during assay system precision and method precision study was within 2.0 and the \%RSD for area of impurity-A, impurity-B, and impurity-C in related compounds method 


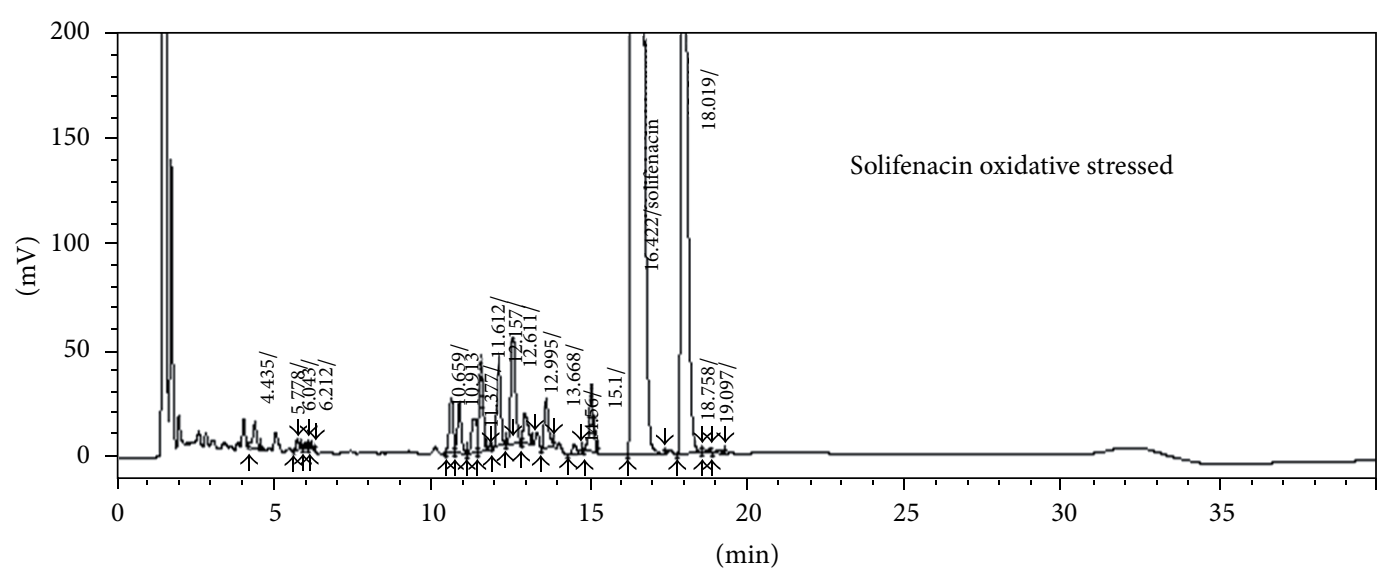

FIgURE 8: Typical HPLC chromatogram of oxidative $\left(10 \% \mathrm{H}_{2} \mathrm{O}_{2}\right)$ stressed solution.

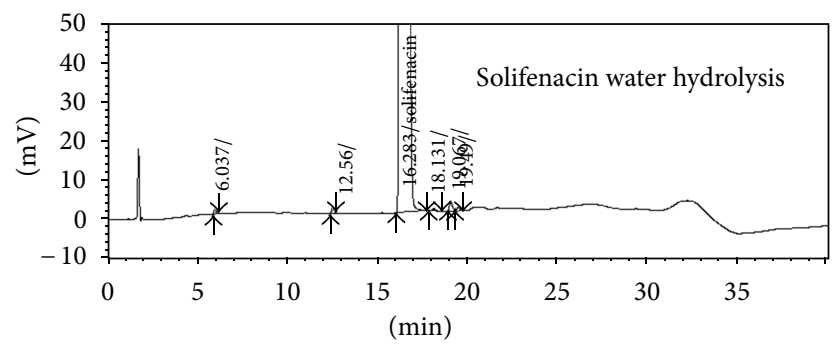

FIGURE 9: Typical HPLC chromatogram of water stressed solution.

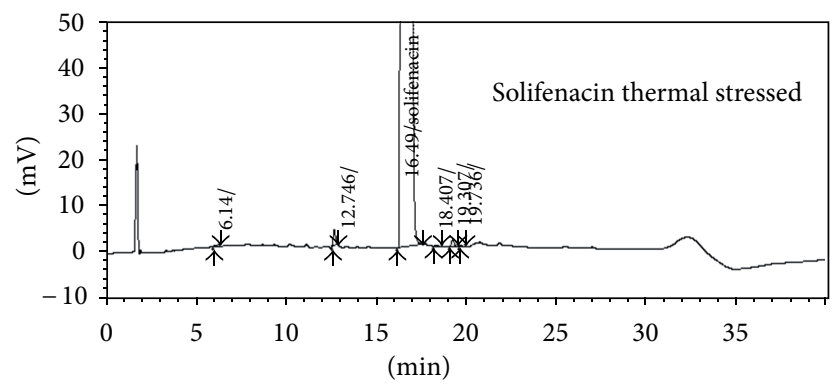

Figure 10: Typical HPLC chromatogram of thermal (Heat at $80^{\circ} \mathrm{C}$ ) stressed solution.

precision study was within 5.0. The \%RSD of assay results obtained in intermediate precision study was within 2.0 and the \%RSD for area of impurity-A, impurity-B, and impurity$\mathrm{C}$ was well within 10.0 confirmed good precision of the method.

3.2.2. Limit of Detection and Limit of Quantification. The limit of detection of solifenacin, impurity-A, impurity-B, and impurity- $\mathrm{C}$ was $0.0064 \%, 0.0034 \%, 0.0025 \%$, and $0.0068 \%$ (analyte concentration, i.e., $0.5 \mathrm{mg} / \mathrm{mL}$ ) for $10 \mu \mathrm{L}$ injection volume. The limit of quantification of solifenacin, impurity$\mathrm{A}$, impurity-B, and impurity-C was $0.02 \%, 0.01 \%, 0.01 \%$, and $0.02 \%$ (analyte concentration, i.e., $0.5 \mathrm{mg} / \mathrm{mL}$ ) for $10 \mu \mathrm{L}$

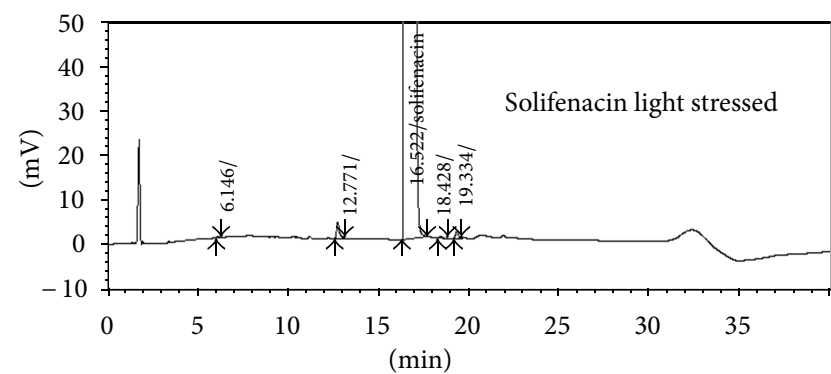

FIgURE 11: Typical HPLC chromatogram of UV stressed solution.

injection volume. The precision at LOQ concentration for impurity-A, impurity-B, and impurity-C was below $10.0 \%$. The accuracy/recovery experiments were carried out for impurity- $\mathrm{A}$, impurity- $\mathrm{B}$, and impurity- $\mathrm{C}$ at LOQ concentration in triplicate and the recoveries were found to be well within $80-115 \%$, which demonstrates that the developed method can accurately measure the impurities present in solifenacin succinate.

3.2.3. Linearity. Linear calibration plot for assay method was obtained over the calibration ranges tested, that is, $0.08-0.12 \mathrm{mg} / \mathrm{mL}$ and the correlation coefficient obtained was greater than 0.999 . The percentage of RSD values for each level is within 2.0 and \%Y-intercept of the calibration curve is -0.3 (within \pm 2 ). These results show that an excellent correlation existed between the peak area and concentration of the analyte.

Linearity calibration plot for related compounds method was obtained over the calibration ranges tested, that is, LOQ, $0.075 \%, 0.12 \%, 0.135 \%, 0.15 \%, 0.18 \%$, and $0.225 \%$ for impurity-A, impurity-B, and impurity-C. The correlation coefficient obtained was greater than 0.995. The percentage of RSD values for each level are within the limit $(<5.0)$. The \%Y-intercept of the calibration curves is $-1.03,-1.36$, and -1.52 for impurity- $A$, impurity-B, and impurity- $\mathrm{C}$, respectively. These results show that an excellent 


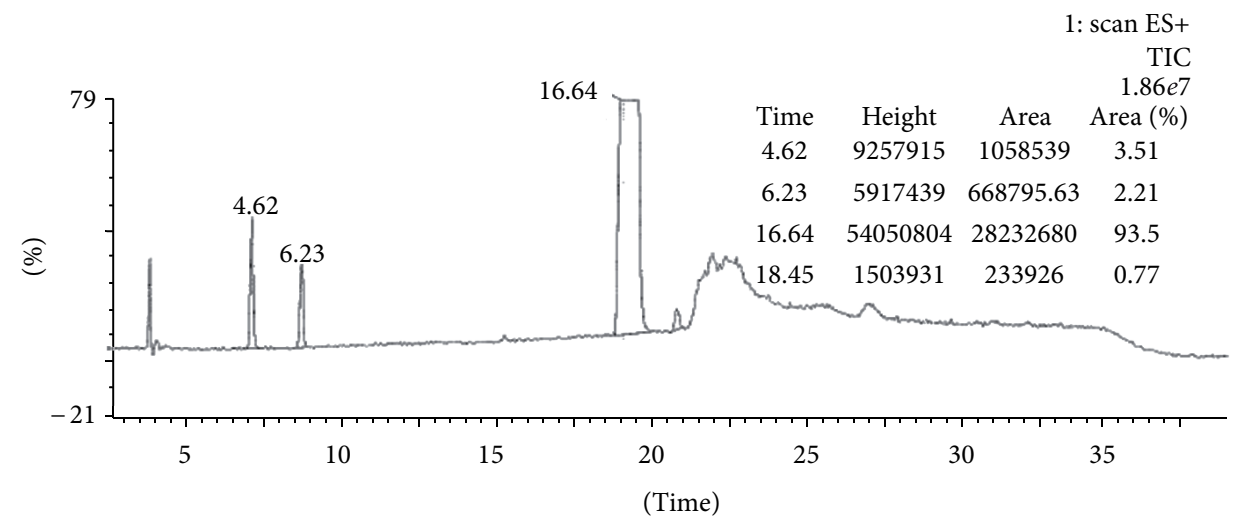

FIGURE 12: Typical LC-MS chromatogram of solifenacin succinate spiked with known standards of impurity-A, impurity-B, and impurity-C.

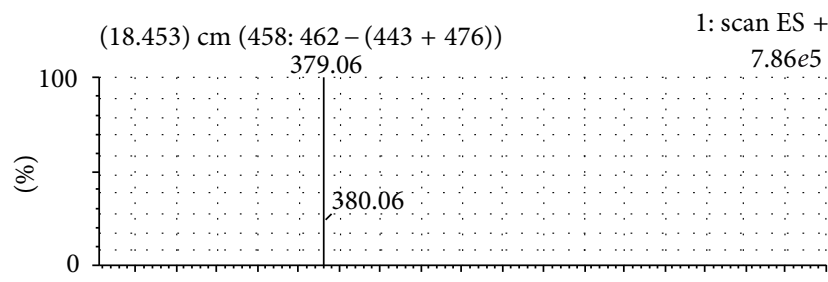

FIGURE 13: MS spectrum of impurity-C at RRT 1.1.

correlation existed between the peak area and concentration of the impurity-A, impurity-B, and impurity-C.

3.2.4. Accuracy. The percentage recovery of solifenacin succinate in assay method is within the limit (98-102). The percentage recovery of impurity- $\mathrm{A}$, impurity- $\mathrm{B}$, and impurity- $\mathrm{C}$ in related compounds method are within the limit $(85 \%$ to $115 \%)$ and recovery data shown for both methods in Table 5. HPLC chromatograms of blank, pure solifenacin succinate, solifenacin succinate spiked with $0.15 \%$ of each impurity and solifenacin succinate assay chromatograms are shown in Figures 2, 3, 4, and 5 .

3.2.5. Robustness. In all the deliberate varied chromatographic conditions carried out as per Section 2.6.5 (flow rate, $\mathrm{pH}$, and column temperature), the resolution between closely eluting impurities (Table 6), namely, impurity-A and impurity- $\mathrm{B}$, solifenacin and impurity- $\mathrm{C}$ was greater than 3.0, illustrating the robustness of the method.

3.2.6. Solution Stability and Mobile Phase Stability. The $\%$ Recovery for assay of solifenacin succinate during solution stability and mobile phase stability experiments was within limit (98-102\%). No significant changes were observed in the content of impurity- $\mathrm{A}$, impurity- $\mathrm{B}$, and impurity- $\mathrm{C}$ during solution stability and mobile phase experiments when performed using related compounds method. The variation content (\%) of each impurity obtained from fresh sample and each time interval of the solution stability should be $\pm 0.03 \%$.
The solution stability and mobile phase stability experiments data confirm that sample solutions and mobile phase used during assay (up to 24 hours) and related compounds determination were stable up to 48 hours.

\section{Results of Forced Degradation Studies}

Significant degradation was not observed in solifenacin succinate stressed sample that was subjected to light, heat, acid, base, and water hydrolysis (Figures 6, 7, 9, 10 and 11). The degradation of drug substance was observed under oxidative conditions (Figure 8). Solifenacin Succinate was degraded into impurity-C during oxidative $\left(10 \% \mathrm{H}_{2} \mathrm{O}_{2}\right.$ after $1 \mathrm{hr}$ treatment). The same was confirmed by coinjection with a qualified impurity-C standard and identified by LCMS. Peak purity test results derived from photo diode array detector, confirmed by that solifenacin succinate peak, are homogeneous and pure in all the analyzed stress samples. The mass balance of samples (Table 7) was closed to $99.5 \%$. The \%assay of solifenacin succinate is unaffected in the presence of impurity-A, impurity-B, impurity-C, and its degradation products confirm the stability indicating power of the developed method.

\section{Impurities Identification by LC-MS}

For identification of impurity-A, impurity-B, and impurityC, the ESI mass spectrum of impurities eluted at RRT 0.28 and 0.37 (Figure 12) in positive ion mode showed a molecular ion peaks at $m / z 208,210\left[(\mathrm{mH})^{+}\right]$, indicating the molecular weights of these compounds are 207 and 209, respectively. From this data we conclude that these two are intermediates, which were used in manufacturing process of solifenacin succinate and these two were also confirmed with photo diode array detector by comparing the spectra's with known standards. These two impurities further were characterized by UV, FT-IR, Mass, and NMR $\left({ }^{13} \mathrm{C},{ }^{1} \mathrm{H}\right)$.

ESI mass spectrum of major impurity formed during the oxidative stress condition at RRT 1.10 in positive ion mode showed a molecular ion peak at $\mathrm{m} / z 379\left[(\mathrm{mH})^{+}\right]$indicating 
the molecular weight of the compound as 378 (Figure 13). This molecular ion mass was 16 mass units higher than that of solifenacin and this indicates the probability of the formation of N-oxide. The same impurity was also formed in base degradation,but in smaller quantities. Further this impurity was synthesized and characterized by UV, FT-IR, Mass, and $\operatorname{NMR}\left({ }^{13} \mathrm{C},{ }^{1} \mathrm{H}\right)$.

\section{Conclusions}

New, accurate, and selective gradient RP-LC methods were proposed for the determination of related compounds and assay of solifenacin succinate drug substance and validated as per ICH guidelines. The method has higher sensitivity for the determination of related compounds than previously reported methods. The method was found to be simple, selective, precise, accurate, and robust. The method was completely validated showing satisfactory data for all the method parameters tested. The developed method is stability indicating and can be used for routine analysis of production samples and also to check the stability of bulk samples of solifenacin succinate.

\section{Acknowledgments}

The authors wish to thank the management of Hetero Research Foundation for their support, encouragement, and permitting this work to communicate for publication. Authors also wish to thank the chemical research and analytical research departments for their cooperation.

\section{References}

[1] A. J. Wein, "Pharmacological agents for the treatment of urinary incontinence due to overactive bladder," Expert Opinion on Investigational Drugs, vol. 10, no. 1, pp. 65-83, 2001

[2] K. Ikeda, S. Kobayashi, M. Suzuki et al., "M3 receptor antagonism by the novel antimuscarinic agent solifenacin in the urinary bladder and salivary gland," Naunyn-Schmiedeberg's Archives of Pharmacology, vol. 366, no. 2, pp. 97-103, 2002.

[3] M. C. Michel, M. Oelke, and N. Zinner, "Novel muscarinic antagonists to treat incontinence and/or overactive bladder," Drug Discovery Today, vol. 2, no. 1, pp. 1-6, 2005.

[4] H. N. Mistri, A. G. Jangid, A. Pudage, D. M. Rathod, and P. S. Shrivastav, "Highly sensitive and rapid LC-ESI-MS/MS method for the simultaneous quantification of uroselective $\alpha_{1}$ blocker, alfuzosin and an antimuscarinic agent, solifenacin in human plasma," Journal of Chromatography B, vol. 876, no. 2, pp. 236-244, 2008.

[5] J. Macek, P. Ptáček, and J. Klíma, "Determination of solifenacin in human plasma by liquid chromatography-tandem mass spectrometry," Journal of Chromatography B, vol. 878, no. 31, pp. 3327-3330, 2010.

[6] T. Yanagihara, T. Aoki, Y. Soeishi, T. Iwatsubo, and H. Kamimura, "Determination of solifenacin succinate, a novel muscarinic receptor antagonist, and its major metabolite in rat plasma by semi-micro high performance liquid chromatography," Journal of Chromatography B, vol. 859, no. 2, pp. 241-245, 2007.
[7] S. Radha Krishna, B. M. Rao, and N. Someswara Rao, "A validated rapid stability-indicating method for the determination of related substances in Solifenacin Succinate by ultra-fast liquid chromatography," Journal of Chromatographic Science, vol. 48, no. 10, pp. 807-810, 2010.

[8] B. S. Landge, A. S. Jadhav, B. S. Dahale et al., "Development and validation of new chromatographic method for the determination of enantiomeric and diastereomeric purity of solifenacin succinate: an anti-muscarinic agent," Chromatography Research International, vol. 2011, Article ID 243491, 7 pages, 2011.

[9] D. Desai, G. Patel, N. Shukla, and S. Rajput, "Development and validation of stability-indicating HPLC method for solifenacin succinate: isolation and identification of major base degradation product," Acta Chromatographica, vol. 24, no. 3, pp. 399-418, 2012.

[10] N. Desai, S. S. Hussen, S. G. Vasanthraju, A. Karthik, and N. Udupa, "Development \& validation of stability indicating HPLC method for determination of Solifenacin in bulk formulations," International Journal of Pharmacy and Pharmaceutical Sciences, vol. 3, no. 1, pp. 70-74, 2011.

[11] L. Singh and S. Nanda, "Spectrophotometric estimation of solifenacin succinate in tablet formulations," Pharmaceutical Methods, vol. 2, no. 1, pp. 21-24, 2011.

[12] R. Seetharaman, K. S. Lakshmi, D. K. Boggavarappu, G. Hariprasad, and K. R. Ravi, "Determination of Balofloxacin in pharmaceutical formulation by zero, first and second order derivative spectrophotometric methods," International Journal of Research in Pharmaceutical Sciences, vol. 2, no. 3, pp. 1052-1057, 2011.

[13] J. D. Desai, G. Patel, D. Ruikar, A. R. Jain, and J. S. Rajput, "Development and validation of stability-indicating HPTLC method of solifenacin succinate," Asian Journal of Pharmaceutical and Biological Research, vol. 1, no. 3, pp. 310-316, 2011.

[14] ICH Guideline Q3A(R2), impurities in New Drug Substances, 2006.

[15] ICH Guideline Q7A, Good Manufacturing Practice Guide for Active Pharmaceutical Ingredients, 2005.

[16] ICH Q2(R1) Validation of Analytical Procedures, Text and Methodology, 2005.

[17] ICH Q1A(R2), "Stability testing of new drug substances and products," in International Conference on Harmonization, 2003.

[18] ICH, "Stability testing of new drugs substances and products (Q1AR)," in International Conference on Harmonization (IFPMA '00), Geneva, Switzerland, 2000.

[19] "ICH draft guidelines on validation of analytical procedures: definitions and terminology, federal Register," in International Conference on Harmonization (IFPMA '95), p. 11260, Geneva, Switzerland, 1995. 

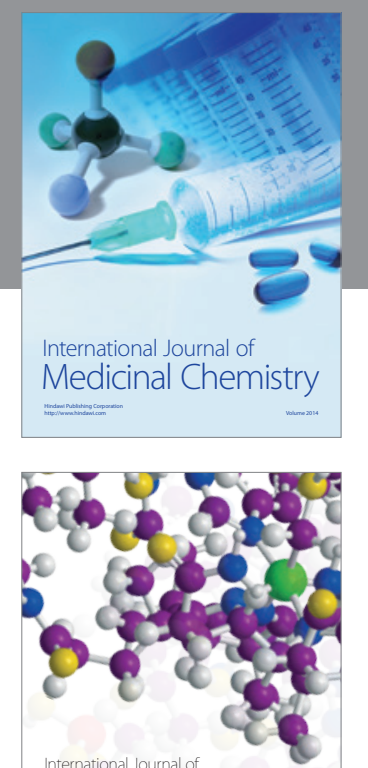

\section{Carbohydrate} Chemistry

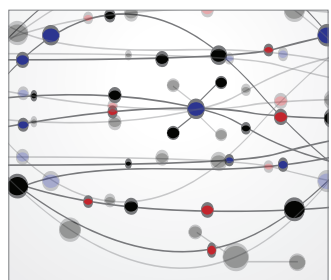

The Scientific World Journal
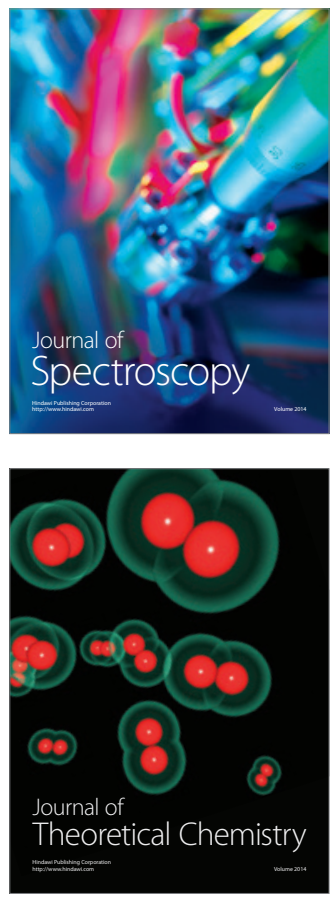
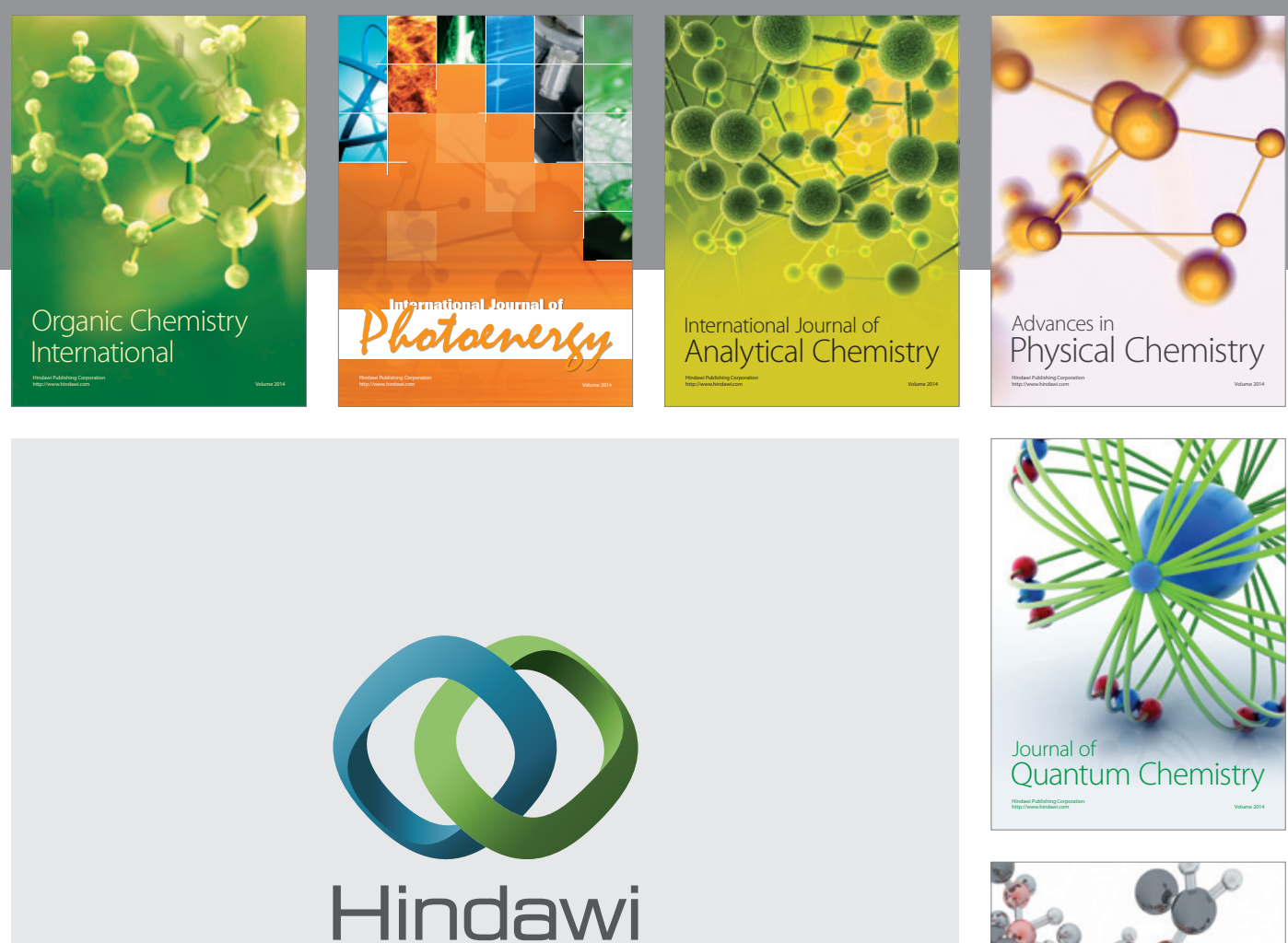

Submit your manuscripts at

http://www.hindawi.com

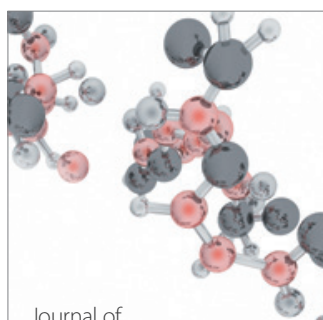

Analytical Methods

in Chemistry

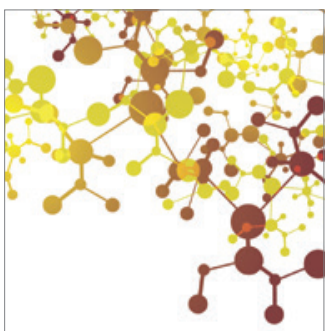

Journal of

Applied Chemistry

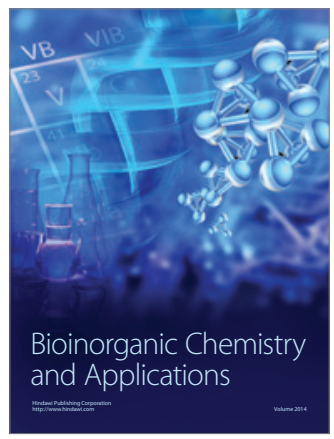

Inorganic Chemistry
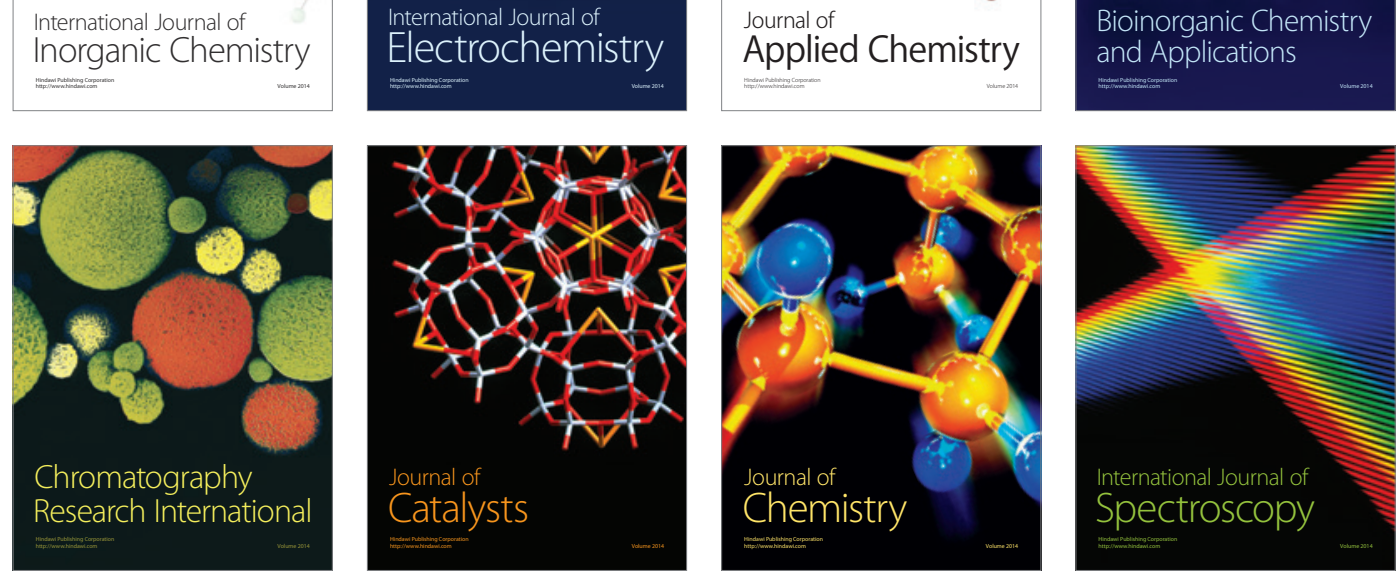